\title{
Avifauna associada a um trecho urbano do Rio Sorocaba, Sudeste do Brasil
}

\author{
Bruna Botti Cruz ${ }^{1,3}$ \& Augusto João Piratelli ${ }^{2}$ \\ ${ }^{1}$ Programa de Pós-graduação em Diversidade Biológica e Conservação, \\ Centro de Ciências e Tecnologias para a Sustentabilidade - CCTS, \\ Universidade Federal de São Carlos - UFSCar, Campus Sorocaba, \\ Rod. João Leme dos Santos, Km 110, CEP 18052-780, Sorocaba, SP, Brasil \\ ${ }^{2}$ Departamento de Ciências Ambientais, Centro de Ciências e Tecnologias para a Sustentabilidade - CCTS, \\ Universidade Federal de São Carlos - UFSCar, Campus Sorocaba, \\ Rod. João Leme dos Santos, Km 110, CEP 18052-780, Sorocaba, SP, Brasil \\ ${ }^{3}$ Autor para correspondência: Bruna Botti Cruz, e-mail: brunabotti@yahoo.com.br
}

CRUZ, B.B. \& PIRATELLI, A.J. Avifauna associated to an urban extend of the Sorocaba River, Southeastern Brazil. Biota Neotrop. 11(4): http://www.biotaneotropica.org.br/v11n4/en/abstract?inventory+bn02411042011

\begin{abstract}
This study aimed to characterize the avifauna associated to the Sorocaba River, a typical urban river in Southeastern Brazil. It crosses the city having the same name, along which different human activities are carried out as recreation and fishing. There, several bird species might find sites for resting, feeding and nesting. We surveyed birds weekly, from September 2008 to September 2009, by 16 point counts (A1 to A16). The quantitative survey registered 65 species (32 Passeres and 33 non-Passeres), and the most well-represented family was Tyrannidae ( $\mathrm{n}=9$ ). The most frequent species were Egretta thula, Amazonetta brasiliensis, Vanellus chilensis, Pitangus sulphuratus, Ardea alba and Pygochelidon cyanoleuca $(\mathrm{FO}=100 \%)$. The higher abundance index values were 46.5 (1626 contacts) for Pygochelidon cyanoleuca, 14.4 (500 contacts) for Phalacrocorax brasilianus and 12.9 (452 contacts) for Amazonetta brasiliensis. The most representative trophic categories were insectivores $(n=26)$, piscivores $(n=11)$ and omnivores $(n=10)$. The cluster analysis revealed the existence of three very similar pairs of points (100 and $95 \%$ of information remaining). Conspicuous differences in the composition of bird fauna along the studied area were detected, probably due to its environmental characteristics and the degree of human disturbance. Moreover, significant differences were observed between the sightings of dry and wet seasons, probably due to the reproductive and migratory activities and an increased availability of food resources that is supposed to happen in the wet season. In this sense, conservation practices should provide the maintenance of habitat heterogeneity, recovering the riparian forests, beaches and marshes, and an effective planning of human activities in their surroundings as well.
\end{abstract}

Keywords: abundance, community structure, points count, temporal variation, urban birds.

CRUZ, B.B. \& PIRATELLI, A.J. Avifauna associada a um trecho urbano do rio Sorocaba, Sudeste do Brasil. Biota Neotrop. 11(4): http://www.biotaneotropica.org.br/v11n4/pt/abstract?inventory+bn02411042011

Resumo: Este estudo teve como objetivo caracterizar a avifauna associada ao rio Sorocaba, um típico rio urbano, do sudeste do Brasil. Ele atravessa a cidade com o mesmo nome, ao longo do qual, várias atividades humanas são realizadas como recreação e pesca. Lá, várias espécies de aves devem encontrar locais para descanso, alimentação e nidificação. As aves foram observadas semanalmente, de setembro de 2008 a setembro de 2009 por meio de 16 pontos fixos (A1 a A16). O levantamento quantitativo registrou 65 espécies (32 passeriformes e 33 não - passeriformes), sendo que a família mais bem representada foi Tyrannidae $(\mathrm{n}=9)$. As espécies mais freqüentes foram Egretta thula, Amazonetta brasiliensis, Vanellus chilensis, Pitangus sulphuratus, Ardea alba e Pygochelidon cyanoleuca $(\mathrm{FO}=100 \%)$. Os maiores valores do índice pontual de abundância foram 46,5 (1626 contatos) para Pgochelidon cyanoleuca, 14,4 (500 contatos) para Phalacrocorax brasilianus e 12,9 (452 contatos) para Amazonetta brasiliensis. As categorias tróficas mais representativas foram dos insetívoros $(\mathrm{n}=26)$, piscívoros $(\mathrm{n}=11)$ e onívoros $(\mathrm{n}=10)$. A análise de agrupamento revelou a existência de três pares de pontos muito semelhantes (100 e 95\% semelhança). Diferenças conspícuas na composição da fauna de aves ao longo da área estudada foram detectadas, provavelmente devido às suas características ambientais e do grau de perturbação humana. Além disso, diferenças significativas nos avistamentos foram observadas entre as estações de seca e chuva, provavelmente em função das atividades reprodutivas e migratórias e do aumento na disponibilidade de recursos alimentares, que supostamente ocorrem no período chuvoso. Neste sentido, práticas conservacionistas devem permitir a manutenção a heterogeneidade de habitats, recuperação da vegetação ripária, praias e banhados, assim como o planejamento efetivo das atividades humanas em seu entorno.

Palavras-chave: abundância, , estrutura de comunidades, pontos de observação, variação temporal, aves urbanas. 


\section{Introdução}

O rápido processo de urbanização se tornou uma das maiores preocupações para a biologia da conservação (Miller \& Hobbs 2002). Isto está associado ao fato de que, em ambientes urbanos a redução de hábitas naturais promove depleção na diversidade de aves, assim como a homogeneização das comunidades, uma vez que poucas espécies são adaptadas para viver nestas áreas de intensa descaracterização (Jokimäki et al. 1996). Além disso, nestes ambientes, diversos fatores interferem na sobrevivência da avifauna, dentre eles as alterações de hábitats devido às mudanças na composição florística e fragmentação da vegetação nativa (Marzluff 2001, Chace \& Walsh 2006), poluição (Eeva et al. 2000), flutuações nas populações de predadores (Sorace 2002), disponibilidade de alimentos, doenças, alterações das condições climáticas (Haggard 1990) e aumento das perturbações, incluindo os níveis de ruídos (Fuller et al. 2007).

Diante do desafio que o progresso urbano representa atualmente para a conservação (Scherer et al. 2006), estudos que exploram padrões de diversidade de aves em diferentes gradientes de urbanização estão se tornando comuns (Blair 1996, Germaine et al. 1998, Willis 2000, Sandström et al. 2006, Lin et al. 2008, Caula et al. 2008).

O desenvolvimento de estudos buscando investigar o papel dos ambientes aquáticos, virtuais "refúgios" para a fauna que persiste no meio urbano, é fundamental, destacando-se a avifauna como potencial medida de qualidade dos hábitats urbanos (Chace \& Walsh 2006), tanto na avaliação da qualidade dos ecossistemas como no registro e monitoramento de alterações provocadas nos ambientes nos quais as malhas hídricas estão inseridas.

Ao longo da extensão do rio Sorocaba, que é o maior afluente da margem esquerda do rio Tietê, encontra-se grande número de aves residentes e migratórias (Regalado 2007). Estas comunidades são reguladas pela capacidade biótica deste ambiente e pelo grau de preservação dos ambientes associados (Branco 2007, Caula et al. 2008). Apesar de seu papel fundamental para a manutenção da biodiversidade de aves, o rio Sorocaba e seus tributários vêm sofrendo impactos ambientais em decorrência do lançamento de esgoto doméstico e industrial, desmatamento da vegetação ciliar, assoreamento, mineração, além da irrigação e uso indiscriminado de agrotóxicos (Smith et al. 2005, Comitê... 2006). Neste contexto, o presente estudo teve por objetivos caracterizar a avifauna associada a um trecho urbano do rio Sorocaba, indicando possíveis ações para a manutenção e aumento da complexidade de suas comunidades.

\section{Material e Métodos}

\section{1. Área de estudo}

O trabalho foi desenvolvido no município de Sorocaba, sudeste paulista, cuja área urbana corresponde a $349,2 \mathrm{~km}^{2}$ ocupada por 580.655 habitantes (Instituto... 2010). As observações foram feitas ao longo de um trecho urbano do rio Sorocaba de 3,01 km (23⒉ 29' 57' S e $47^{\circ} 27^{\prime} 25^{\prime}$ ' O), margeado por uma importante via de trânsito e por uma ciclovia (Figura 1). O rio Sorocaba nasce no planalto de Ibiúna, no lado Oeste da Serra do Mar, a aproximadamente $900 \mathrm{~m}$ de altitude, entrando na pré-serra, em curso acidentado, sendo formado pelos rios Sorocabaçu, Sorocamirim e Una. Deságua no rio Tietê em sua porção média superior, no município de Laranjal Paulista, possuindo $180 \mathrm{~km}$ em linha reta e $227 \mathrm{~km}$ considerando seu leito em trajeto natural e área de drenagem de $5.269 \mathrm{~km}^{2}$ (Smith et al. 2005).

O clima da região é tropical úmido-seco ou de savana, Cwa baseado na classificação de Köppen segundo Ayoade (2006), sendo marcadas uma estação seca (abril a setembro), com baixas precipitações, atingindo $33,6 \mathrm{~mm}$ no mês de julho e média térmica de $13{ }^{\circ} \mathrm{C}$, e chuvosa (outubro a março), quando se registram os maiores índices pluviométricos, chegando a $222,3 \mathrm{~mm}$ no mês de janeiro, e médias de temperatura de $26^{\circ} \mathrm{C}$ (Canabarro et al. 2008).

Ao longo de seu percurso urbano o rio apresenta uma série de características distintas, com diferentes perfis e graus de perturbação. Entre elas destacam-se áreas com remanescentes de mata ciliar, proximidade com a via principal de trânsito, áreas de recreação e lazer como praças e ciclovia, presença de tubulações para saída de água pluvial e de afluentes.

O município está inserido em um ecótono, na zona de contato entre as formações savana (cerrado) e floresta ombrófila (Instituto... 2009), o que lhe confere características vegetacionais, climáticas e faunísticas das respectivas formações, proporcionando grande valor ecológico (Montes 2005, Comitê... 2006).

\section{Coleta de dados}

Para o levantamento quantitativo da comunidade de aves foi empregada metodologia de pontos de contagem fixos (Blondel et al. 1981, adaptada por Vielliard \& Silva 1990). Dezesseis pontosforam marcados, respeitando uma distância mínima de 200 m entre os pontos, buscando-se amostrar características ambientais distintas no trecho estudado.

Estes pontos foram visitados semanalmente, em média cinco pontos por semana, durante treze meses, de setembro de 2008 a setembro de 2009, totalizando 35 dias de observação, pela manhã (das 6 ás 8 horas) ou à tarde (das 17 às 18 horas). Em cada ponto, permaneceuse 10 minutos, sempre na margem direita do rio, anotando-se todas as detecções visuais e auditivas com distância ilimitada das aves presentes nas margens, no rio e em vôo (Donatelli et al. 2004). Os registros visuais foram realizados com auxílio de binóculo $(8 \times 40 \mathrm{~mm})$ e a identificação das espécies com apoio de literatura quando necessário (Höfling \& Camargo 1999, Efe et al. 2001, Regalado 2007). Vocalizações também foram utilizadas como registro das espécies e para identificações posteriores foram realizadas consultas em portais eletrônicos que possuem bancos de registros sonoros como Xeno-canto (Planqué \& Vellinga 2010).

As espécies foram agrupadas em categorias tróficas conforme Willis (1979), Motta-Junior (1990), Sick (1997) e Curcino et al. (2007), além de observações pessoais. Quando não houvesse coincidência entre o item alimentar da espécie entre esses autores, assumiu-se a categoria adotada na publicação mais recente. A nomenclatura das espécies seguiu o Comitê Brasileiro de Registros Ornitológicos (2011).

\section{Análise de dados}

Para a análise dos dados obtidos, o Índice Pontual de Abundância (IPA) (Vielliard \& Silva 1990) de cada espécie foi calculado a partir do número de contatos com a espécie, dividido pelo número total de pontos amostrados. A Frequência de Ocorrência (FO) foi obtida pela razão entre o número de dias de registro de cada espécie e o número total de dias em que o estudo foi realizado (Vielliard \& Silva 1990, Oliveira 1995, Lima \& Fontana 2000). Por meio dos valores de FO as espécies foram classificadas como residentes (R) quando $\mathrm{FO} \geq 60 \%$, prováveis residentes $(\mathrm{P})$ para $60 \%>\mathrm{FO} \geq 15 \%$ e ocasionais e/ou sobrevoantes $(\mathrm{O})$ para FO $<15 \%$ (Vielliard \& Silva 1990, Almeida et al. 1999, Mendonça-Lima \& Fontana 2000).

Diferenças significativas na abundância entre as estações seca e chuvosa foram investigadas utilizando-se o teste de chi-quadrado ( $\alpha=0.05$ ) (Gotelli \& Ellison 2011). Para estimar a diversidade sazonal de espécies foi aplicado o índice de diversidade de ShannonWiener (H'), com logarítmo natural (Krebs 1998) e a riqueza de Margalef (Magurran 2004) para as duas estações do ano. Foi 

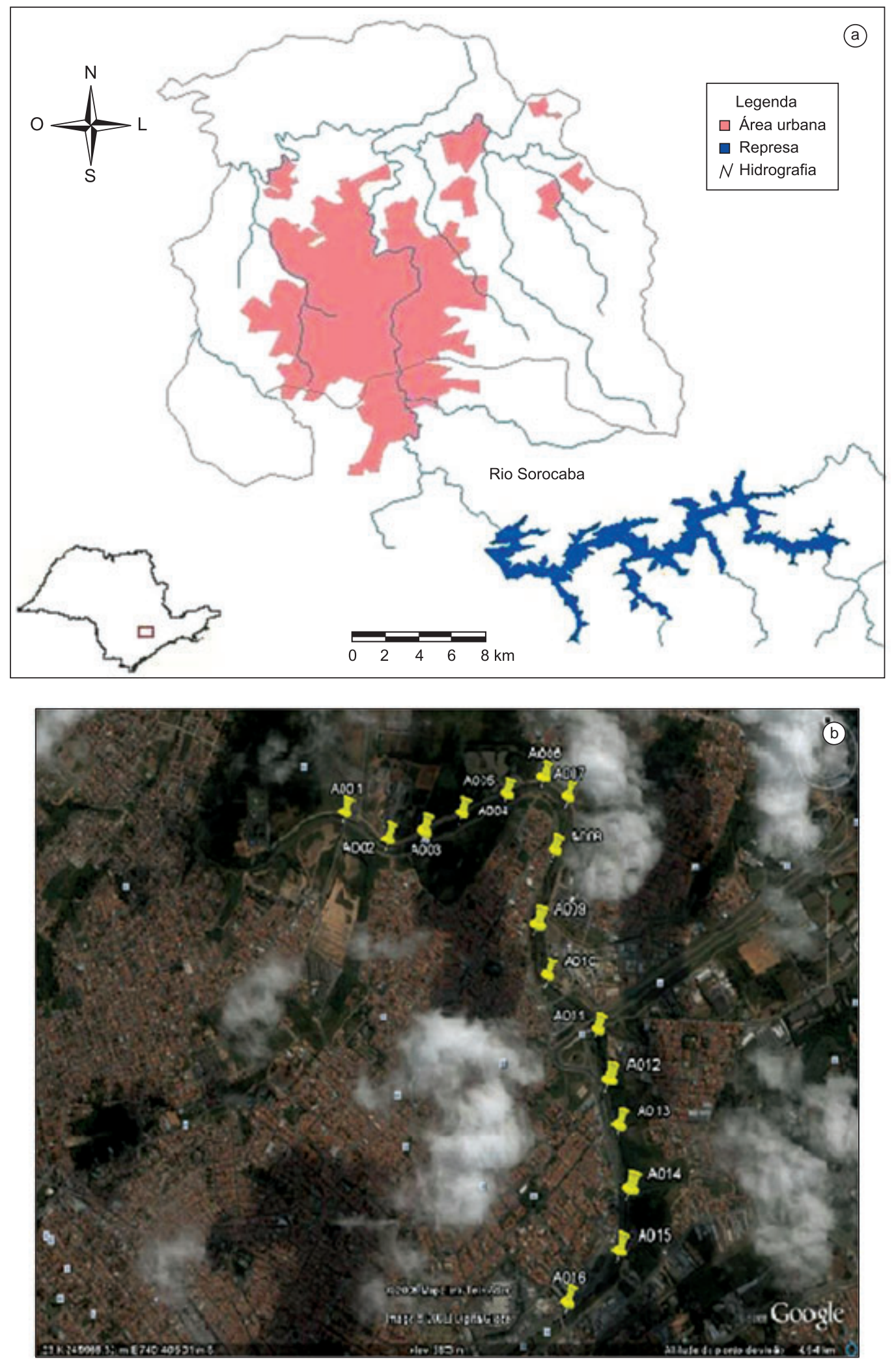

Figura 1. a) Localização do rio Sorocaba e do município de Sorocaba; e b) dos 16 pontos fixos marcados ao longo de trecho urbano deste rio. Adaptado de Centro de Referência em Informação Ambiental (2005) e Google Earth.

Figure 1. a) Location of the Sorocaba River and the city of Sorocaba; and b) the 16 fixed points marked along an urban section of this river. Adapted from Reference Center on Environmental Information (2005) and Google Earth. 
utilizado o método "bootstrap" para estimar o intervalo de confiança a 95\% (Magurran 2004). Tanto o teste de qui-quadrado, quando os estimadores ecológicos foram calculados com o auxílio do software PAST (Hammer et al. 2001).

Para avaliar padrões espaciais da avifauna foi realizada uma análise de agrupamento entre os pontos de amostragem, por meio da distância de Bray-Curtis e " $\beta$-flexible como método de associação $(\beta=0.25)$. Bray-Curtis é considerado o melhor estimador de distância para gradientes heterogêneos como o ambiente estudado e desconsidera duplas ausências na comunidade (McCune \& Grace 2002). Para esta análise, o número de registros de cada espécie foi considerado. Foi utilizado o programa PC-ORD 4.0 (McCune \& Mefford 1997).

\section{Resultados}

\section{Composição da avifauna}

Foram registradas 65 espécies, representando 15 ordens e 34 famílias, das quais 33 são não-Passeriformes e 32 Passeriformes (Tabela 1). As famílias mais representativas quanto à riqueza de espécies foram Tyrannidae $(n=9)$, Ardeidae $(n=6)$ e Hirundinidae $(n=4)$. Houve tendência à estabilização na curva de acúmulo de espécies a partir da $11^{\mathrm{a}}$ amostra, o que sugere que o esforço amostral ( 35 coletas) foi suficiente para caracterizar a avifauna do rio Sorocaba no trecho estudado (Figura 2).

Aves residentes $(n=15)$ e prováveis residentes $(n=34)$ representaram $75,4 \%$ das aves registradas. Ao menos 10 espécies (15,4\% da avifauna amostrada) apresentaram possíveis movimentos migratórios sazonais (outubro a março) (Tabela 1), possivelmente utilizando o rio como ponto de passagem ou permanência temporária. Algumas delas inclusive reproduziram-se na área, como: Dendrocyna viduata, Himantopus melanurus, Tyrannus melancholicus e Tyrannus savana, cujos ninhos ou imaturos foram avistados. Além destas, no mesmo período, também foram observadas atividades reprodutivas de Anthus lutescens, Tachycineta albiventer, Amazonetta brasiliensis,
Vanellus chilensis, Ardea alba, Gallinula chloropus, Jacana jacana, Patagioenas picazuro, Fluvicola nengeta e Pitangus sulphuratus, pela detecção de ninhos e/ou imaturos.

Os valores máximos do Índice Pontual de Abundância (IPA) foram 107,4 (1.718 contatos) para Pygochelidon cyanoleuca, 14,4 (500 contatos) para Phalacrocorax brasilianus, 13,8 (221) para Himantopus melanurus e 12,9 (452 contatos) para Amazonetta brasiliensis. Para 12 dos pontos monitorados, a espécie Pygochelidon cyanoleuca obteve os valores máximos do IPA.

Segundo a frequência de ocorrência das espécies, as categorias de aves residentes $(15,23 \%)$ e prováveis residentes $(34,52 \%)$ representaram $75,3 \%$ da avifauna registrada, enquanto $16(24,6 \%)$ foram consideradas ocasionais ou sobrevoantes (Tabela 1).

Em relação aos hábitos alimentares, houve predominância de espécies que consomem principalmente insetos 41,5\% ( $\mathrm{n}=27)$. (Figura 4). Frutos são itens predominantes somente para cinco $(9,23 \%)$ espécies encontradas: Elaenia flavogaster, Euphonia chlorotica, Brotogeris chiriri, Tangara cayana e Thraupis sayaca (Willis 1979, Motta-Junior 1990, Sick 1997 e Curcino et al. 2007).

\section{Estrutura da comunidade}

Foram registrados 8.705 contatos no total (média de 564 contatos/mês). Nos meses de dezembro de 2008 a maio de 2009 foi constatado maior número de contatos, enquanto que entre junho a setembro de 2009 obteve-se o menor número de contatos. A riqueza foi menor nos meses de maio a junho de 2009, assim como a diversidade, cuja diferença mensal, entretanto não foi significativa.

Em relação à variação mensal nos avistamentos totais e na diversidade de espécies no período de estudo, houve um acentuado e significativo aumento dos avistamentos totais na estação chuvosa, (outubro a março), $\left(\chi^{2}=93,57, \mathrm{p}<0,05\right)$ (Figura 3). O índice de diversidade de Shannon-Wiener (H') e seu respectivo intervalo de confiança, apresentou valor 3,02 (2,97 -3,05) para a estação de seca e 3,04 (2,99-3,07) para a chuvosa, porém a diferença não foi

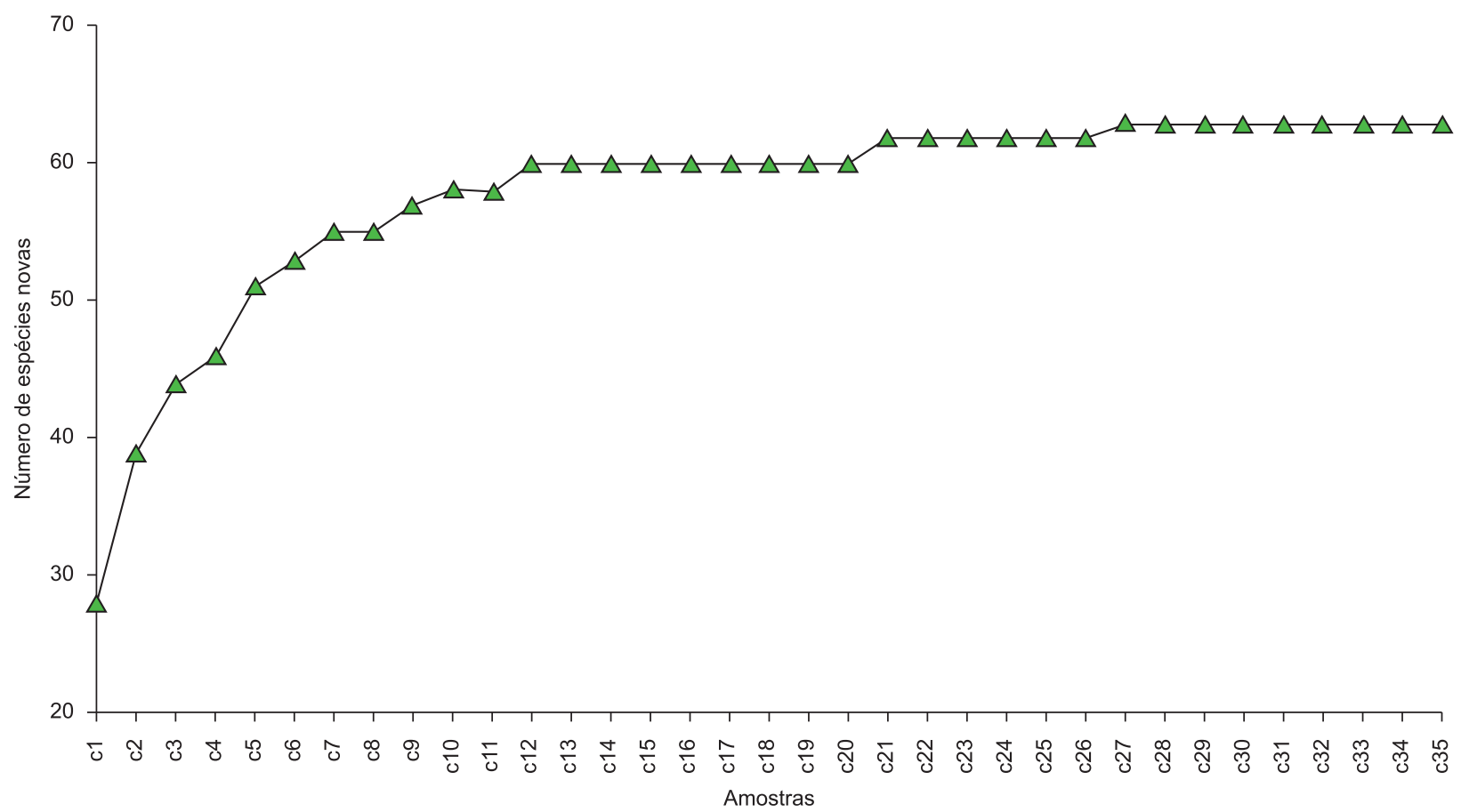

Figura 2. Curva de acúmulo de espécies de aves registradas em um trecho urbano do rio Sorocaba entre setembro de 2008 e setembro de 2009.

Figure 2. Accumulative curve of new bird species recorded in an urban section of the Sorocaba river between September 2008 and September 2009. 
Tabela 1. Espécies registradas nos 16 pontos fixos em trecho urbano do rio Sorocaba. Classificação das espécies observadas segundo a freqüência de ocorrência e categorias tróficas segundo Willis (1979). $\mathrm{FO}=$ frequência de ocorrência da espécie; $\mathrm{N}_{\mathrm{di}}=$ total de dias em que a espécie foi registrada; $\mathrm{R}=$ categoria residente; $\mathrm{P}=$ categoria provável residente; $\mathrm{O}=$ categoria ocasional ou sobrevoante. Status migratório definido quando apresentando algum tipo de deslocamento/migração na área, segundo observações próprias e Motta-Junior et al. (2008). Dados coletados entre setembro de 2008 e setembro de 2009.

Table 1. Species recorded in the 16 fixed points in an urban section of the Sorocaba River. Observed species classified by frequency of occurrence and trophic groups (Willis 1979). $\mathrm{FO}=$ frequency of occurrence; $\mathrm{N}_{\mathrm{di}}=$ total days in which the species was recorded, $\mathrm{R}=$ resident category, $\mathrm{P}=$ probable resident category; $\mathrm{O}=$ occasional category. Migratory status defined as having any level of displacement/migration in the area, according to personal observations and MottaJunior et al. (2008). Data collected between September 2008 and September 2009.

\begin{tabular}{|c|c|c|c|c|c|c|c|}
\hline Família & Espécie & Total de contatos & Guilda trófica & $\mathbf{N}_{\mathrm{di}}$ & FO $(\%)$ & Categoria & Migração \\
\hline \multirow[t]{2}{*}{ Anatidae } & Amazonetta brasiliensis & 293 & Foliófago & 35 & 100,0 & $\mathrm{R}$ & - \\
\hline & Dendrocyna viduata & 192 & Foliófago & 22 & 62,9 & $\mathrm{R}$ & M \\
\hline Anhingidae & Anhinga anhinga & 24 & Piscívoro & 13 & 37,1 & $\mathrm{P}$ & - \\
\hline Phalacocoracidae & Phalacrocorax brasilianus & 500 & Piscívoro & 33 & 94,3 & $\mathrm{R}$ & - \\
\hline \multirow[t]{6}{*}{ Ardeidae } & Ardea alba & 433 & Piscívoro & 34 & 97,1 & $\mathrm{R}$ & - \\
\hline & Ardea cocoi & 21 & Piscívoro & 17 & 48,6 & $\mathrm{P}$ & - \\
\hline & Butorides striata & 94 & Piscívoro & 29 & 82,9 & $\mathrm{R}$ & - \\
\hline & Egretta thula & 259 & Piscívoro & 32 & 91,4 & $\mathrm{R}$ & - \\
\hline & Nycticorax nycticorax & 119 & Piscívoro & 31 & 88,6 & $\mathrm{R}$ & - \\
\hline & Syrigma sibilatrix & 2 & Insetívoro & 2 & 5,7 & $\mathrm{O}$ & - \\
\hline Ciconiidae & Mycteria americana & 1 & Piscívoro & 1 & 2,9 & $\mathrm{O}$ & $M^{*}$ \\
\hline Threskiornithidae & Platalea ajaja & 53 & Malacófago & 11 & 31,4 & $\mathrm{P}$ & M \\
\hline Carthartidae & Coragyps atratus & 27 & Detritívoro & 9 & 25,7 & $\mathrm{P}$ & - \\
\hline Accipitridae & Rupornis magnirostris & 10 & Carnívoro & 6 & 17,1 & $\mathrm{P}$ & - \\
\hline Falconidae & Polyborus plancus & 38 & Onívoro & 13 & 37,1 & $\mathrm{P}$ & - \\
\hline \multirow[t]{2}{*}{ Rallidae } & Aramides cajanea & 12 & Onívoro & 7 & 20,0 & $\mathrm{P}$ & - \\
\hline & Gallinula chloropus & 289 & Onívoro & 34 & 97,1 & $\mathrm{R}$ & - \\
\hline Charadriidae & Vanellus chilensis & 368 & Insetívoro & 35 & 100,0 & $\mathrm{R}$ & - \\
\hline Recurcirostridae & Himantopus melanurus & 290 & Insetívoro & 20 & 57,1 & $\mathrm{P}$ & M \\
\hline Jacanidae & Jacana jacana & 76 & Insetívoro & 18 & 51,4 & $\mathrm{P}$ & - \\
\hline \multirow[t]{4}{*}{ Columbidae } & Columbina talpacoti & 61 & Granívoro & 13 & 37,1 & $\mathrm{P}$ & - \\
\hline & Columba livia & 9 & Onívoro & 5 & 14,3 & $\mathrm{O}$ & - \\
\hline & Zenaida auriculata & 12 & Granívoro & 5 & 14,3 & $\mathrm{O}$ & - \\
\hline & Patagioenas picazuro & 405 & Frugívoro & 28 & 80,0 & $\mathrm{R}$ & - \\
\hline Psittacidae & Brotogeris chiri & 7 & Frugívoro & 3 & 8,6 & $\mathrm{O}$ & - \\
\hline \multirow[t]{2}{*}{ Cuculidae } & Crotophaga ani & 117 & Insetívoro & 17 & 48,6 & $\mathrm{P}$ & - \\
\hline & Guira guira & 52 & Insetívoro & 9 & 25,7 & $\mathrm{P}$ & - \\
\hline Apodidae & Streptoprocne zonaris & 7 & Insetívoro & 2 & 5,7 & $\mathrm{O}$ & - \\
\hline Trochilidae & Eupetomena macroura & 18 & Nectarívoro & 11 & 31,4 & $\mathrm{P}$ & - \\
\hline \multirow[t]{3}{*}{ Alcedinidae } & Megaceryle torquata & 37 & Piscívoro & 20 & 57,1 & $\mathrm{P}$ & - \\
\hline & Chloroceryle amazona & 10 & Piscívoro & 10 & 28,6 & $\mathrm{P}$ & - \\
\hline & Chloroceryle americana & 9 & Piscívoro & 8 & 22,9 & $\mathrm{P}$ & - \\
\hline \multirow[t]{2}{*}{ Picidae } & Colaptes campestris & 11 & Insetívoro & 5 & 14,3 & $\mathrm{O}$ & - \\
\hline & Dryocopus lineatus & 1 & Insetívoro & 1 & 2,9 & $\mathrm{O}$ & - \\
\hline \multirow[t]{3}{*}{ Furnariidae } & Furnarius rufus & 22 & Insetívoro & 9 & 25,7 & $\mathrm{P}$ & - \\
\hline & Certhiaxis cinnamomeus & 13 & Insetívoro & 6 & 17,1 & $\mathrm{P}$ & - \\
\hline & Synallaxis albescens & 1 & Insetívoro & 1 & 2,9 & $\mathrm{O}$ & - \\
\hline Thamnophilidae & Thamnophilus caerulescens & 1 & Insetívoro & 1 & 2,9 & $\mathrm{O}$ & - \\
\hline \multirow[t]{3}{*}{ Tyrannidae } & Todirostrum cinereum & 13 & Insetívoro & 11 & 31,4 & $\mathrm{P}$ & - \\
\hline & Elaenia flavogaster & 7 & Insetívoro & 7 & 20,0 & $\mathrm{P}$ & - \\
\hline & Pyrocephalus rubinus & 1 & Insetívoro & 1 & 2,9 & $\mathrm{O}$ & $M^{*}$ \\
\hline
\end{tabular}


Cruz, B.B. \& Piratelli, A.J.

Tabela 1. Continuação...

\begin{tabular}{|c|c|c|c|c|c|c|c|}
\hline Família & Espécie & Total de contatos & Guilda trófica & $\mathbf{N}_{\mathrm{di}}$ & FO $(\%)$ & Categoria & Migração \\
\hline \multirow{6}{*}{ Tyrannidae } & Fluvicola nengeta & 154 & Insetívoro & 34 & 97,1 & $\mathrm{R}$ & - \\
\hline & Arundinicola leucocephala & 2 & Insetívoro & 2 & 5,7 & $\mathrm{O}$ & - \\
\hline & Machetornis rixosa & 41 & Insetívoro & 12 & 34,3 & $\mathrm{P}$ & - \\
\hline & Pitangus sulphuratus & 281 & Insetívoro & 35 & 100,0 & $\mathrm{R}$ & - \\
\hline & Tyrannus melancholicus & 77 & Insetívoro & 21 & 60,0 & $\mathrm{P}$ & $\mathrm{M}$ \\
\hline & Tyrannus savana & 45 & Insetívoro & 12 & 34,3 & $\mathrm{P}$ & M \\
\hline \multirow[t]{4}{*}{ Hirundinidae } & Stelgidopteryx ruficollis & 60 & Insetívoro & 9 & 25,7 & $\mathrm{P}$ & M \\
\hline & Progne tapera & 22 & Insetívoro & 7 & 20,0 & $\mathrm{P}$ & M \\
\hline & Pygochelidon cyanoleuca & 1722 & Insetívoro & 53 & 94,3 & $\mathrm{R}$ & - \\
\hline & Tachycineta albiventer & 4 & Insetívoro & 3 & 8,6 & $\mathrm{O}$ & - \\
\hline Troglodytidae & Troglodytes musculus & 24 & Onívoro & 10 & 28,6 & $\mathrm{P}$ & - \\
\hline Mimidae & Mimus saturninus & 4 & Onívoro & 3 & 8,6 & $\mathrm{O}$ & - \\
\hline Turdidae & Turdus leucomelas & 12 & Onívoro & 7 & 20,0 & $\mathrm{P}$ & - \\
\hline Motacillidae & Anthus lutescens & 24 & Insetívoro & 8 & 22,9 & $\mathrm{P}$ & - \\
\hline \multirow[t]{3}{*}{ Thraupidae } & Thraupis palmarum & 3 & Frugívoro & 2 & 5,7 & $\mathrm{O}$ & - \\
\hline & Thraupis sayaca & 76 & Onívoro & 24 & 68,6 & $\mathrm{R}$ & - \\
\hline & Tangara cayana & 2 & Frugívoro & 2 & 5,7 & $\mathrm{O}$ & - \\
\hline \multirow[t]{2}{*}{ Emberezidae } & Ammodramus humeralis & 46 & Granívoro & 13 & 37,1 & $\mathrm{P}$ & - \\
\hline & Volatina jacarina & 19 & Granívoro & 11 & 31,4 & $\mathrm{P}$ & - \\
\hline \multirow[t]{2}{*}{ Ictaridae } & Chrysomus ruficapillus & 92 & Granívoro & 7 & 20,0 & $\mathrm{P}$ & M \\
\hline & Molothrus bonariensis & 144 & Onívoro & 10 & 28,6 & $\mathrm{P}$ & - \\
\hline Frigilidae & Euphonia chlorotica & 11 & Frugívoro & 8 & 22,9 & $\mathrm{P}$ & - \\
\hline Estrildidae & Estrilda astrild & 144 & Granívoro & 11 & 31,4 & $\mathrm{P}$ & - \\
\hline Passeridae & Passer domesticus & 103 & Onívoro & 22 & 62,9 & $\mathrm{R}$ & - \\
\hline
\end{tabular}

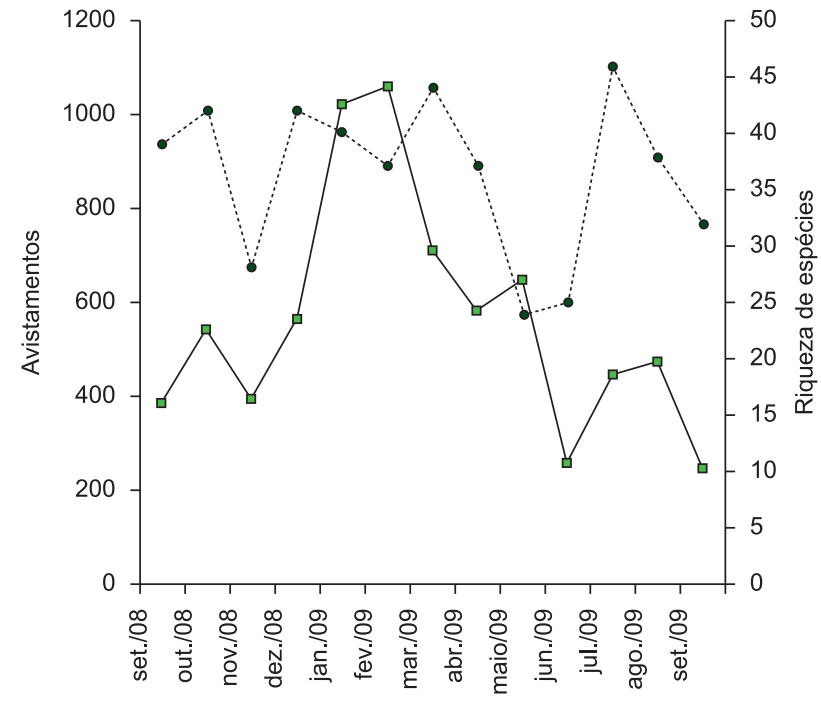

Meses de coletas

$\neg-$ Registros totais mensais $\quad \ldots \bullet$ Riqueza mensal

Figura 3. Totais de avistamentos e riqueza de espécies obtidos em relação aos meses de observação no trecho urbano do rio Sorocaba entre setembro de 2008 e setembro de 2009.

Figure 3. Total bird sightings and species richness from between September 2008 and September 2009 in an urban section of the Sorocaba River.

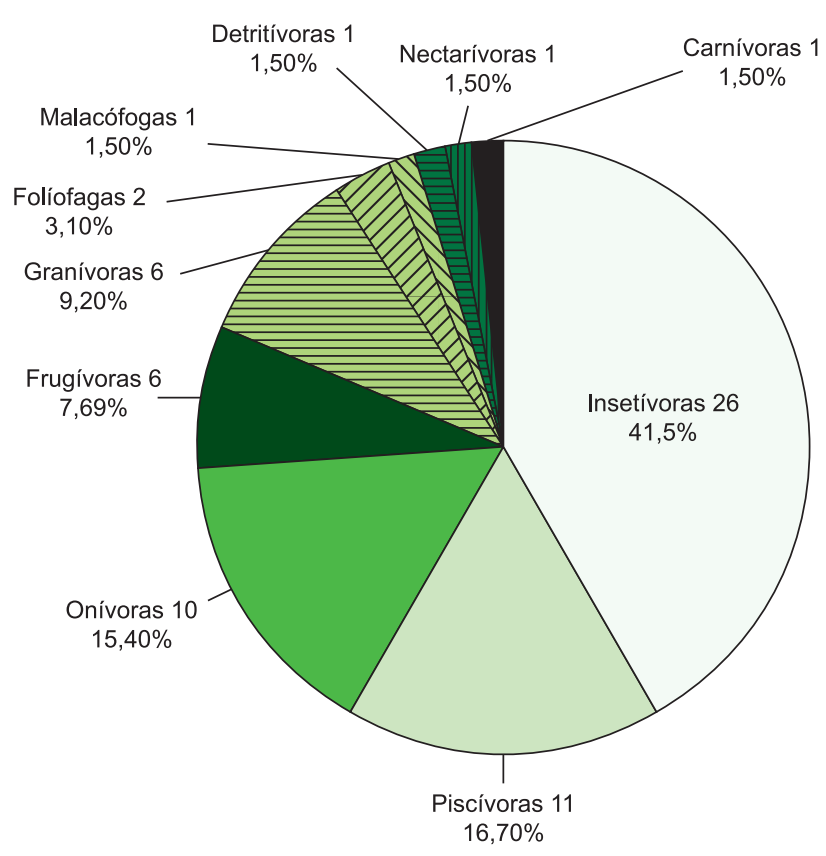

Figura 4. Proporção de espécies nas categorias de alimentação determinadas para as aves do trecho estudado do rio Sorocaba.

Figure 4. Proportion of bird species in trophic groups in an urban section of the Sorocaba River. 


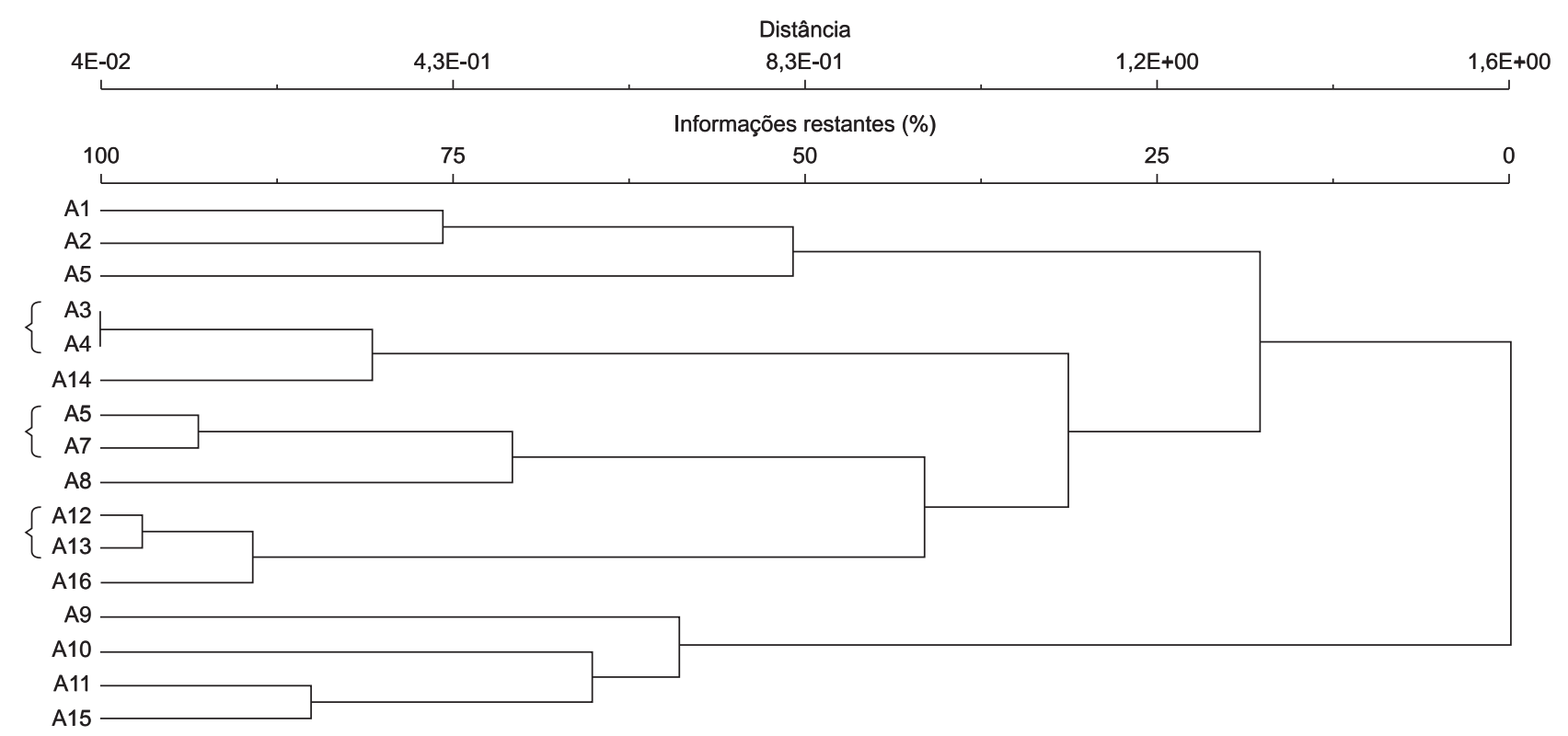

Figura 5. Análise de cluster baseada na abundância e riqueza de espécies para os 12 pontos monitorados do trecho urbano do rio Sorocaba, Sorocaba, SP, com base na distância de Bray-Curtis e o método de associação flexible- $\beta(\beta=-0,250)$.

Figure 5. Cluster analysis based on abundance and species richness for the 16 monitored points of an urban section of the Sorocaba River, based on Bray-Curtis distance and flexible- $\beta$ association method $(\beta=-0.250)$.

significativa, assim como a riqueza de Margalef que foi de 7,46 $(6,72-7,46)$ na seca e $6,93(6,20-6,93)$ na estação chuvosa.

A análise de agrupamento formou seis grupos principais, com semelhança acima de $75 \%$ entre os pontos de amostragem. Os três pares que obtiveram maior valor de semelhança foram A3 e A4 (100\%), A12 e A13 (95\%) e A5 e A7 (90\%) de semelhança (Figura 5).

\section{Discussão}

\section{Composição da avifauna}

Sessenta e cinco espécies foram registradas no presente trabalho, o que corresponde a 23,8\% das 273 espécies mencionadas por Regalado (2007) para a cidade de Sorocaba e região. Em relação às observações no rio Sorocaba, $90 \%$ das 20 aves aquáticas encontradas por este autor foram observadas no presente estudo, e nós acrescentamos Amazonetta brasilienses, que não havia sido registrada neste local. Em um dos poucos trabalhos realizados na área urbana de Sorocaba, Silva \& Nakano (2008) observaram 33 espécies (44\% das aqui amostradas) em um remanescente de cerrado próximo a um bairro urbano. Petry \& Scherer (2008) amostraram aves no rio dos Sinos (RS), tendo encontrado 65 espécies, das quais $31(47,7 \%)$ coincidem com as aqui observadas. Alves \& Perreira (1998) observarm 31 espécies de aves na Lagoa Rodrigo de Freitas no Rio de janeiro, das quais $23(74,19 \%)$ coincidem com as apontadas no presente estudo.

A maior representatividade da família Tyrannidae também foi observada por Torga et al. (2007) e Silva \& Nakano (2008), ressaltando a alta frequência desta família nestes ambientes. Piacentini e Campbell-Thompson (2006) e Marini et al. (2009) afirmam que a família Tyrannidae ocupa os mais diversificados ambientes, tendo se adaptado aos mais variados nichos ecológicos, inclusive em ambientes urbanos. Além disso, deve-se destacar que muitas vezes os tiranídeos apresentam preferências por ambientes alterados, como é o caso de Tyrannus savana (Piacentini \& Campbell-Thompson 2006).

No presente estudo houve predomínio de espécies da categoria residentes, o que está de acordo com o padrão verificado por Chace e Walsh (2006), Allen \& O'Conner (2000) e Poague et al. (2000) que destacam os efeitos da urbanização, que tende a favorecer o estabelecimento de espécies residentes à migrantes.

$P$. rubinus, $M$. americana, D. lineatus, T. caerulescens e $S$. albescens foram classificadas como ocasionais ou sobrevoantes com apenas um contato e, portanto podem ser consideradas raras ou pouco comuns na área de estudo. Sua raridade pode estar associada à biologia das espécies, que demandam ambientes mais arborizados requerendo, muitas vezes áreas heterogêneas e menos antropizadas como é o caso das três últimas, que são normalmente encontradas no interior ou borda de matas (Willis 1979).

A família Ardeidae também esteve bem representada, já que contém um dos maiores grupos com características adaptadas às áreas úmidas (Scherer et al. 2006, Branco 2007). A preferência da família por estes ambientes está relacionada ao hábito alimentar e à biologia reprodutiva das espécies; cujos indivíduos se alimentam principalmente de peixes e nidificam frequientemente formando densos ninhais em arbustos situados em zonas ripárias, banhados e manguezais (Sick 1997, Scherer et al. 2006), coforme aqui registrado.

A espécie Pygochelidon cyanoleuca (Hirundinidae) foi caracterizada como residente, uma vez que a espécie foi frequente durante todo o período de estudo, porém, esta categoria não implica que os indivíduos sejam residentes da área de estudo. O grande número de indivíduos de $P$. cyanoleuca está relacionado ao padrão de distribuição agregado da espécie (Pereira \& Melo 2010) e aos hábitos de forrageamento, que consiste na caça ativa em bandos muito numerosos, além de suas atividades migratórias conhecidas para a América do Sul, que podem ter contribuído para o aumento da abundância na estação chuvosa. Conforme Sick (1997) e Nunes e Tomas (2008) a espécie possui populações migrantes e residentes, as quais durante os deslocamentos se misturam temporariamente em áreas propicias para a invernada, como o que pode ter ocorrido durante o período de chuva na área de estudo.

As espécies insetívoras predominaram no presente estudo, seguidas das piscívoras e onívoras. O predomínio de insetívoras e onívoras em áreas urbanas tem sido registrado em diversas cidades brasileiras (Argel-De-Oliveira 1995, Matarazzo-Neuberguer 1995, 
Franchin \& Marçal Júnior 2004, Lin et al. 2008, Lopes \& Anjos 2006, Torga et al. 2007) e já havia sido reportada como um efeito da urbanização por Chace e Walsh (2006) e Allen \& O'Conner (2000). A urbanização tende a favorecer as espécies insetívoras que forrageiam no ar e no solo, como é o caso dos representantes da família Hirundinidae e Tyrannidae, que também foram representativas no trabalho de Torga et al. (2007). Argel-De-Oliveira (1995) destaca que, em regiões urbanizadas predominam espécies com dietas flexíveis como os onívoros e os insetívoros não especializados, sendo que os insetívoros que se alimentam em vôo, que foram mais representativos no presente estudo, exploram um recurso alimentar que não é prejudicado pela urbanização e que, segundo esta última autora, podem se tornar mais abundantes.

Outro tópico que deve ser destacado quanto à categoria dos insetívoros, diz respeito à maior proporção de espécies prováveis residentes e ocasionais, em relação às efetivamente residentes. Isto pode estar relacionado ao grau de especificidade das espécies, que, em sua maioria, provavelmente utilizam o trecho estudado como área complementar de forrageio e nidificação, dependendo apenas parcialmente do rio. Além disso, a baixa proporção de aves frugívoras e nectarívoras nas cidades pode ser resultado da carência de recursos alimentares (Willis 1979). Em projetos paisagísticos urbanos muitas vezes predominam espécies vegetais ornamentais, em detrimento às que produzem frutos carnosos ou flores, o que torna estes ambientes atrativos para espécies generalistas que habitam áreas abertas e que se beneficiam dos recursos disponibilizados pela ação antrópica, como é o caso dos insetívoros, onívoros e granívoros (Argel-De-Oliveira 1995, Torga et al. 2007).

A ausência de complexidade estrutural da vegetação nas áreas urbanas propicia o estabelecimento e permanência constante de espécies que são menos exigentes quanto à oferta de recursos (Crooks et al. 2004), o que pode ter se refletido no padrão de categorias tróficas aqui encontrado. Lin et al. (2008) ao estudarem a influência do ambiente urbano na avifauna de Taipei (Taiwan) também confirmaram a predominância das categorias de insetívoros e onívoros naquelas paisagens.

\section{Estrutura da comunidade}

O aumento dos avistamentos entre os meses de outubro de 2008 a março de 2009 provavelmente está associado à elevação da pluviosidade, conhecido para a região nesta época, com possível aumento na disponibilidade de alimento e coincidência com a estação reprodutiva para a maioria das aves (Sick 1997, Franchin \& Marçal Júnior 2004). O efeito da sazonalidade teve efeito na abundância das espécies, porém não foi significativo para a riqueza e consequentemente para a diversidade no presente estudo. Isto pode estar relacionado à urbanização, que promove a homogeneização dos ambientes naturais, tornando-os menos atrativos para a fauna (Blair 2001, Brand et al. 2008). Estes ambientes favorecem a permanência de espécies generalistas com distribuição agregada e que são frequentes o ano todo, como é o caso das famílias Ardeidae e Hirundinidae.

Estes resultados corroboram com outros estudos encontrados na literatura. Heezik et al. (2008) ao analisarem a diversidade de aves em um gradiente urbano em uma cidade na Nova Zelândia, verificaram que a estrutura, composição e grau de cobertura da vegetação não alteraram a riqueza ou a diversidade, mas sim a estrutura primária das comunidades, no caso a abundância. Nelson e Nelson (2001) também verificaram situação semelhante na área metropolitana de Denver, Estados Unidos.

A análise de agrupamento evidenciou três pares de pontos com maior semelhança (Figura 5), o que pode estar relacionado com a proximidade entre eles e a algumas características ambientais dos mesmos, incluindo ausência de vegetação ciliar e acentuada inclinação das margens (pontos A3 e A4), presença de espécies vegetais arbóreas (A12 e A13), e forte influência antrópica (pontos A5 e A7). A presença de vegetação permitiu o estabelecimento de espécies da família Alcedinidae (Megaceryle torquata, Chloroceryle amazona e Chloroceryle americana). Estas usualmente pousam em árvores, troncos ou galhos secos à beira d'água para pescar, tornando-se, portanto, dependentes destes substratos (Accordi \& Barcellos 2006, Baretta et al. 2006). Nestes ambientes também foi possível observar Aramides cajanea (Rallidae), que utilizou esta vegetação para se refugiar e forragear. A forte presença humana em certas porções da área de estudo, implicando em ruídos acentuados, trânsito de veículos e transeuntes, poderia explicar a presença de espécies mais generalistas como Phalacrocorax brasilianus e Gallinula chloropus, além de certos Ardeidae (Accordi \& Barcellos 2006).

\section{Conclusões}

A comunidade de aves do trecho urbano estudado do rio Sorocaba é constituída especialmente por espécies generalistas, residentes ou prováveis residentes com hábitos insetívoros e onívoros, resultado provavelmente, da perturbação histórica ao ambiente pela intensa interferência antrópica. A sazonalidade não teve efeito significativo na riqueza e diversidade das comunidades, entretanto afetou a abundância, o que pode estar relacionado com a maior disponibilidade de recursos alimentares e também com a utilização da área de estudo para atividades reprodutivas de algumas espécies, que implicam no aumento populacional. Por isso, recomenda-se que as atividades recreativas próximas ao corpo d'água no período de chuva sejam reguladas, uma vez que, a presença humana pode interferir nas atividades desempenhadas pelas espécies. Por fim, um enriquecimento das espécies vegetais ao longo do rio é altamente recomendando, priorizando espécies com frutos carnosos, o que poderia atrair mais espécies frugívoras especializadas e auxiliar na recuperação das funções ecológicas do local.

\section{Agradecimentos}

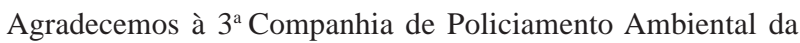
região de Sorocaba pela embarcação utilizada no reconhecimento do trecho amostrado e acompanhamento, e a Luiz Augusto Manfré, pelo apoio nas coletas dados, críticas e sugestões ao trabalho.

\section{Referências Bibliográficas}

AYOADE, J.O. 2006. Introdução à Climatologia para os Trópicos. Editora Bertrand Brasil, Rio de Janeiro,332p.

ACCORDI, I.A. \& BARCELLOS, A. 2006. Composição da avifauna em oito áreas úmidas da Bacia Hidrográfica do Lago Guaíba, Rio Grande do Sul. Rev. Bras. Ornit. 14(2):101-115.

ALLEN, A.P. \& O'CONNER, R.J., 2000. Hierarchical correlates of bird assemblage structure on northeastern USA lakes. Environ. Monit. Assess. 62:15-35. http://dx.doi.org/10.1023/A:1006244932033

ALMEIDA, M.E.C., VIELLIARD, J.M.E. \& DIAS, M.M. 1999. Composição da avifauna em duas matas ciliares da Bacia do Rio Jacaré-Pepira, São Paulo, Brasil. Rev. Bras. Zool. 16(4):1087-1098.

ALVES, M.A.S. \& PEREIRA, E.F. 1998. Richness, abundance and seasonality of bird species in a lagoon of an urban area (Lagoa Rodrigo de Freitas) of Rio de Janeiro, Brazil. Ararajuba 6(2):110-116.

ARGEL-DE-OLIVEIRA, M.M. 1995. Aves e vegetação em um bairro residencial da cidade de São Paulo (São Paulo, Brasil). Rev. Bras. Zool. 12(1):110-116.

BARETTA, L., PETRY, M.V. \& SANDER, M. 2006. Ecological aspects and abundance of kingfishers in the Sinos River, São Leopoldo, RS. Neotrop. Biol. Cons.1(1):42-47. 
BLAIR, R.B. 1996. Land use and avian species diversity along an urban gradient. Ecol. Appl. 6:506-519. http://dx.doi.org/10.2307/2269387

BLAIR, R.B., 2001. Birds and butterflies along urban gradients in two ecoregions of the United States: is urbanization creating a homogeneous fauna? In Biotic Homogenization: The Loss of Diversity Through Invasion and Extinction (J.L. Lockwood \& M.L. McKinney, eds.). Kluwer Academic Publishers, Boston, p.33-56.

BLONDEL, J., FERRY, C. \& FROCHOT, B. 1981. Point counts with unlimited distance. Stud. Avian Biol. (6):414-420.

BRANCO, J.O. 2007. Avifauna aquática do Saco da Fazenda (Itajaí, Santa Catarina, Brasil):uma década de monitoramento. Rev. Bras. Zool. 24(4):873-882. http://dx.doi.org/10.1590/S010181752007000400003

BRAND, L.A., WHITE, G.C. \& NOON, B.R. 2008. Factors influencing species richness and community composition of breeding birds in a desert riparian corridor. The Condor 110(2):199-210. http://dx.doi.org/10.1525/ cond.2008.8421

CANABARRO, L., TOLEDO, M.T. \& BARELLA, W. 2008. Peixes do Rio Piragibu-Mirim em Sorocaba-SP. Rev. Eletron. Biol. 1(3):31-49.

CAULA, S., MARTY, P. \& MARTIN, J.L. 2008. Seasonal variation in species composition of an urban bird community in Mediterranean France. Landsc. Urb. Plan. 87:1-9. http://dx.doi.org/10.1016/j. landurbplan.2008.03.006

CENTRO DE REFERÊNCIA EM INFORMAÇÃO AMBIENTAL CRIA. 2004. Mapa de vegetação remanescente do Estado de São Paulo. Programa Biota/Fapesp, Instituto Florestal/SMA, Atlas Biota. www. sinbiota.cria.org.br (último acesso em 02/10/2010).

CHACE, J.F. \& WALSH, J.J., 2006. Urban effects on native avifauna: a review. Landsc. Urb. Plan. 74:46-69. http://dx.doi.org/10.1016/j. landurbplan.2004.08.007

COMITÊ DE BACIAS HIDROGRÁFICAS. 2006. Nossas Águas. Comitê de Bacias Hidrográficas dos rios Sorocaba e Médio Tietê, Sorocaba.

COMITÊ BRASILEIRO DE REGISTROS ORNITOLÓGICOS CBRO. 2011. Lista das aves do Brasil do Comitê Brasileiro de Registros Ornitológicos. http://www.cbro.org.br/CBRO/listabr.htm (último acesso em 26/09/2010).

CROOKS, K.R., SUAREZ, A.V. \& BOLGER, D.T. 2004. Avian assemblages along a gradient of urbanization in a highly fragmented landscape. Biol. Conserv..115:451-462.http://dx.doi.org/10.1016/S0006-3207(03)00162-9

CURCINO, A., SANT'ANA, C.E.R. \& HEMING, N.M. 2007. Comparação de três comunidades de aves na região de Niquelândia, GO. Rev. Bras. Ornit.15(4):574-584.

DONATELLI, R.J., COSTA, T.V.V. \& FERREIRA, C.D. 2004. Dinâmica da avifauna em fragmentos de mata na Fazenda Rio Claro, Lençóis Paulista, São Paulo, Brasil. Rev. Bras. Zool. São Paulo, 21:97-114.

EEVA, T., TANHUANPÄ̈̈, S., RABERGH, C., AIRAKSINEN, S., NIKINMAA, M. \& LEHIKOINEN, E. 2000. Biomarkers and fluctuating asymmetry as indicators of pollution-induced stress in two hole-nesting passerines. Func. Ecol. 14: 235-243. http://dx.doi.org/10.1046/j.13652435.2000.00406.x

EFE, M.A., MOHR, L.V. \& BUGONI, L. 2001. Guia Ilustrado das Aves dos Parques de Porto Alegre. PROAVES, SMAM, COPESUL, CEMAVE, Porto Alegre, Brasil.

FRANCHIN, A.G. \& MARÇAL JÚNIOR, O. 2004. A riqueza da avifauna no Parque Municipal do Sabiá, zona urbana de Uberlândia (MG). Biotemas 17(1):179-202.

FULLER, R.A., WARREN, P.H. \& GASTON, K.J. 2007. Daytime noise predicts nocturnal singing in urban robins. Biol. Lett. 3:368 370. PMid:17456449. PMCid:2390663. http://dx.doi.org/10.1098/ rsbl.2007.0134

GERMAINE, S.S., ROSENSTOCK, S.S., SCHWEINSBURG, R.E. \& RICHARDSON, W.S. 1998. Relationships among breeding birds, habitat, and residential development in greater Tucson, Arizona. Ecol. Appl. 8(3):680-691. http://dx.doi.org/10.1890/10510761(1998)008[0680:RABBHA]2.0.CO;2
GOTELLI, N.J. \& ELLISON, A.M. 2011. Princípios de estatística em ecologia. Artmed, Porto Alegre.

HAGGARD, W.H. 1990. Urban weather. Int. J. Environ. Stud. 36:73-82. http://dx.doi.org/10.1080/00207239008710584

HAMMER, Ø., HARPER, D.A.T. \& RYAN, P.D. 2001. PAST: Paleontological Statistics Software Package for Education and Data Analysis. Palaeont. Electron. 1(4):9p.

HEEZIK, Y., LUDWIG, K., WHITWELL, S.\& MCLEAN, I.G. 2008. Nest survival of birds in an urban environment in New Zealand. N. Z. J. Ecol.. 32(2):155-165.

HÖFLING, E. \& CAMARGO, H.F.A. 1999. Aves no Campus. Edusp, São Paulo, Brasil.

INSTITUTO BRASILEIRO DE GEOGRAFIA E ESTATÍSTICA - IBGE. IBGE-Cidades@: São Paulo. Sorocaba. http://www.ibge.gov.br/ cidadesat/topwindow.htm?1 (último acesso em 18/10/2011).

INSTITUTO FLORESTAL. 2009. Centro de Referência em Informação Ambiental. Mapa de Vegetação Remanescente do Estado de São Paulo. Programa Biota, Fapesp.

JOKIMÄKI, J., SUHONEN, J., INKI, K. \& JOKINEN, S. 1996. Biogeographical comparison of winter bird assemblages in urban environments in Finland. J. Biogeog. 23:379-386. http://dx.doi. org/10.1046/j.1365-2699.1996.00033.x

KREBS, C.J. 1998. Ecological Methodology. 2nd ed. Harper \&Row, New York, 518p.

LIMA, A.M. \& FONTANA, C.S. 2000. Composição, freqüência e aspectos biológicos da avifauna no Porto Alegre Country Clube, Rio Grande do Sul. Ararajuba 8(1):1-8.

LIN, Y.B., LIN, Y.P. \& FANG, W.T. 2008. Mapping and assessing spatial multiscale variations of birds associated with urban environments in metropolitan Taipei, Taiwan. Environ. Monit. Assess.145:209-226. PMid:18193333. http://dx.doi.org/10.1007/s10661-007-0030-7

LOPES, E.V. \& ANJOS, L. 2006. A composição da avifauna do campus da Universidade Estadual de Londrina, norte do Paraná, Brasil. Rev. Bras. Zool. 23(1):145-156. http://dx.doi.org/10.1590/S010181752006000100006

MAGURRAN, A.E. 2004. Measuring biological diversity. Blackwell Science, Oxford.

MARINI, M. A., LOBO, Y., LOPES, L. E., FRANÇA, L. F. \& PAIVA, L. V. 2009. Biologia reprodutiva de Tyrannus savana (Aves, Tyrannidae) em cerrado do Brasil Central. Biota Neotrop. 9(1): http://www. biotaneotropica.org.br/v9n1/pt/fullpaper?bn01009012009+pt (último acesso em 21/01/2010).

MARZLUFF, J.M. 2001. Worldwide urbanization and its effects on birds. In Avian Ecology and Conservation in an Urbanizing World: 19-48 (J.M. Marzluff, R. Bowman \& R. Donelly,eds). Kluwer, New York.

MATARAZZO-NEUBERGUER, W.M. 1995. Comunidade de cinco parques e praças da Grande São Paulo, estado de São Paulo. Ararajuba 3:13-19.

MCCUNE, B. \& GRACE, J.B. 2002. Nonmetric Multidimensional Scaling. In Analysis of Ecological Communities. MJM, Software, Oregon, 125p.

MCCUNE, B. \& MEFFORD, M.J. 1997. PC-ORD. Multivariate analysis of ecological data. MJM Software Desing, Gleneden Beach.

MENDONÇA-LIMA, A. \& FONTANA, C.S. 2000. Composição, frequiência e aspectos biológicos da avifauna no Porto Alegre Country Clube, Rio Grande do Sul. Ararajuba 8(1):1-8.

MILLER, J. \& HOBBS, R. 2002. Conservation where people live and work. Conserv. Biol. 16: 330-337. http://dx.doi.org/10.1046/j.15231739.2002.00420.x

MONTES, J. 2005. Fauna de Culicidae da Serra da Cantareira, São Paulo, Brasil. Rev. Saúde Pública 39(4):578-584. http://dx.doi.org/10.1590/ S0034-89102005000400010

MOTTA-JUNIOR, J.C. 1990. Estrutura trófica e composição das avifaunas de três habitats terrestres na região central do estado de São Paulo. Ararajuba 1:65-71. 
MOTTA-JUNIOR, J.C., GRANZINOLLI, M.A.M. \& DEVELEY, P.F. 2008. Aves da estação ecológica de Itirapina, estado de São Paulo, Brasil. Biota Neotrop. 8(3):207-227. http://www.biotaneotropica.org.br/v8n3/en/abstr act?inventory+bn00308032008 (último acesso em 24/03/ 2010).

NELSON, G.S. \& NELSON, S.M. 2001. Bird and butterfly communities associated with two types of urban riparian areas. UrbanEcosyst. 5:95108. http://dx.doi.org/10.1023/A:1022339203875

NUNES, A.P. \& TOMAS, W.M. 2008. Aves migratórias e nômades ocorrentes no Pantanal. Embrapa Pantanal, Corumbá.

OLIVEIRA, M. M. A. 1995. Aves e vegetação em um bairro residencial da cidade de São Paulo (São Paulo, Brasil). Rev. Bras. Zool., 12(1): 81-92.

PEREIRA, Z.P. \& MELO, C. 2010. Nidificação da andorinha azul-ebranca (Pygochelidon cyanoleuca) em ambiente urbano do Sudeste do Brasil. Biotemas 23(3):117-123. http://dx.doi.org/10.5007/21757925.2010v23n3p117

PETRY, M.V. \& SCHERER, J.F.M. 2008. Distribuição da avifauna em um gradiente no rio dos Sinos, São Leopoldo, Rio Grande do Sul, Brasil. Biodiversidade Pampeana 6(2):19-29.

PIACENTINI, V.Q. \& CAMPBELL-THOMPSON, E.R. 2006. Lista comentada da avifauna da microbacia hidrográfica da Lagoa de Ibiraquera, Imbituba, SC. Biotemas 19(2):55-65.

PLANQUÉ, B. \& VELLINGA, W.P. 2010. Xeno-canto Foundation. http:// www.xeno-canto.org/ (último acesso em 02/01/2010).

POAGUE, K.L., JOHNSON, R.J. \& YOUNG, L.J., 2000. Bird use of rural and urban converted railroad rights-of-way in southeast Nebraska. Wildl. Soc. B 28, 852-864.

REGALADO, L.B. 2007. Observando as Aves nas Áreas Verdes de Sorocaba e Região. Sorocaba, Brasil.
SANDSTRÖM, U.G., ANGELSTAM, P. \& MIKUSIÑSKI, G. 2006. Ecological diversity of birds in relation to the structure of urban green space. Landsc. Urb. Plan.77:39-53. http://dx.doi.org/10.1016/j. landurbplan.2005.01.004

SCHERER, J.F.M., SCHERER, A.L., PETRY, M.V. \& TEIXEIRA, E.C. 2006. Estudo da avifauna associada à área úmida situada no Parque Mascarenhas de Moraes, zona urbana de Porto Alegre (RS). Biotemas 19(1):107-110.

SICK, H. 1997. Ornitologia Brasileira. Editora Nova Fronteira, Rio de Janeiro.

SILVA, L.A.C. \& NAKANO, C.A. 2008. Avifauna em uma área de cerrado no bairro do Central Parque, município de Sorocaba, São Paulo, Brasil. Rev. Eletron. Biol. 1(1):54-78.

SMITH, W.S., SALMAZZI, B.A., POSSOMATO, H.M., OLIVEIRA, L.C.A., ALMEIDA, M.A.G., PUPO, R.H. \& TAVARES, T.A. 2005. A bacia do rio Sorocaba: caracterização e principais impactos. Rev. Cient. Imapes 3(3):51-57.

SORACE, A. 2002. High density of bird and pest species in urban habitats and the role of predator abundance. Ornis. Fenn. 79: 60-71.

TORGA, K., FRANCHIN, A.G. \& MARÇAL JÚNIOR, O. 2007. A avifauna em uma seção da área urbana de Uberlândia, MG. Biotemas 20(1):7-17.

VIELLIARD, J.M.E. \& SILVA, W.R. 1990. Nova Metodologia de levantamento quantitativo e primeiros resultados no interior de São Paulo. In IV Encontro Nacional dos Anilhadores. Recife, p.117-151.

WILLIS, E.O. 1979. The composition of avian communities in remanescent woodlots in southern Brazil. Pap. Avulsos Zool. 33(1):1-25.

WILLIS, E.O. 2000. Ranking urban avifaunas (Aves) by number of localities per species in São Paulo, Brazil. Iheringia Sér. Zool. 88:139-146.

Recebido em 17/02/2011

Versão reformulada recebida em 01/11/2011

Publicado em 02/12/2011 